\title{
UNE NOUVELLE FILAIRE « DE LABORATOIRE »(*)
}

\author{
Par M. BALtazARD, A.-G. ChabaUd, Ch. MOFIDI et A. Minou
}

Nous avons précédemment publié (2) les premiers renseignements obtenus sur le cycle évolutif d'une nouvelle filaire : Dipetalonema blanci Chabaud 1952 (3). La suite des recherches que nous poursuivons sera publiée ultérieurement, mais il apparaît déjà que, parmi toutes les espèces utilisées pour les recherches sur les filarioses, celle-ci offre de telles commodités que nous nous devons de la mettre dès à présent à la disposition des laboratoires intéressés.

L'hôte naturel de Dipetalonema blanci est un Mérion : Meriones libycus Lichtenstein 1823, et son vecteur est un petit Ornithodore de terrier : Ornithodorus tartakovskyi Olenev 1931, dont nous avons récemment donné une étude détaillée (1).

Le Meriones libycus est un animal très doux, facile à manipuler et à conserver en captivité, où sa reproduction peut être aisément obtenue $\left({ }^{* *}\right)$; sa répartition géographique est très large et de nombreuses sous-espèces ont été décrites de toute l'Afrique du Nord, du proche et du moyen Orient.

L'Ornithodorus tartakovskyi est très facile à élever et à multiplier au laboratoire, dans les conditions que nous avons décrites (3) et qui sont celles de tous les Ornithodores de terrier : $29^{\circ} \mathrm{C}$., et $60^{\circ}$ hygrométriques au moins. Le cycle complet de l'œuf à l'adulte peut être obtenu dans ces conditions en moins de trois mois. Les Ornithodores sont conservés, sur du papier-filtre froissé, en tubes de verre fermés par un coton peu serré entouré d'une gaze fine. Pour l'élevage et la reproduction, le contenu du tube (Ornithodores et papier) est vidé dans un tube Borrel fermé par une gaze fine, tenue par un caoutchouc, et où l'on a placé un rongeur nouveau-né (rat, souris, lapin). Le rongeur nouveau-né est pratiquement sans défense : les larves ou jeunes nymphes se gorgent en moins d'un quart

(*) Note lue au Congrès de Médecine tropicale et du Paludisme, à Istanbul (septembre 1953).

(**) F. Petter, au Laboratoire de Zoologie des Mammifères du Muséum d'Histcire naturelle de Paris, élève actuellement des M. libycus d'Iran et d'Algérie, et a pu fournir des reproducteurs à un élevage commercial pour la multiplication et l'utilisation par les laboratoires.

ANs, DE PARasitologie, T, XXVIII, $\mathrm{N}^{\circ}$ 5-6, - 1953. 
d'heure, les adultes en une heure environ. Dans les conditions de l'élevage, où l'on cherche la multiplication, le repas des formes jeunes (larves et jeunes nymphes) peut être donné tous les quinze jours ; les jeunes adultes ne peuvent être gorgés plus d'une fois par mois et les gros adultes tous les deux mois.

Par contre, les Ornithodores gardés en réserve pour les expériences, ou dont on veut retarder l'évolution, peuvent être conservés à $15^{\circ} \mathrm{C}$. et $50^{\circ}$ hygrométriques ; dans ces conditions, les repas peuvent être donnés tous les trois mois seulement pour les formes jeunes et une fois par an pour les adultes.

L'expérimentation avec ces Ornithodores sur le Mérion est également fort simple : l'animal (très doux, nous l'avons dit) est attaché sur le dos après rasage de la paroi abdominale. Les Ornithodores sont mis dans une petite cupule métallique rapidement retournée sur l'abdomen et tenue en place par une bague de caoutchouc ceinturant le Mérion. Le plateau sur lequel est attaché l'animal est luimême mis au centre d'un grand plateau émaillé blanc rempli d'eau : précaution contre les évasions. La récolte des Ornithodores est facile, les exemplaires gorgés perdant leur agilité.

Les conditions dans lesquelles Dipetalonema blanci peut être conservée au laboratoire et les possibilités expérimentales offertes par cette filaire peuvent être définies par les quelques données suivantes.

$$
\text { * * * }
$$

Chez l'Ornithodore, l'étude des formes larvaires est aisée, par dissection de l'Acarien sous la loupe binoculaire ou le faible grossissement du microscope dans un verre de montre en eau physiologique.

Il faut, à $29^{\circ}$, vingt jours environ aux microfilaires ingérées par l'Ornithodore pour atteindre le $3^{\circ}$ stade, infestant pour le vertébré. Cette évolution peut être considérablement ralentie par abaissement de la température : à $15^{\circ} \mathrm{C}$., les larves au $3^{\circ}$ stade peuvent être retrouvées vivantes et infestantes après un an. Cette particularité permet de garder sans peine en réserve des Ornithodores infestés ; en effet, comme nous l'avons vu, à cette température, l'Ornithodore peut rester très longtemps sans se nourrir : condition nécessaire, car toute piqûre de l'Ornithodore représente automatiquement la perte au cours du repas de la plus grande partie, sinon de la totalité, de ses larves infestantes.

Une seule précaution, mais capitale, doit être prise dans l'expérimentation avec les Ornithodores. On a toujours tendance à chercher à faire absorber à ceux-ci le maximum de microfilaires pour obtenir 
une infestation plus riche: or, celle-ci est dangereuse pour l'Ornithodore; les lésions multiples causées aux cæcums par le passage de microfilaires trop nombreuses dans la cavité générale entraînent la suffusion du sang ingéré dans tout le corps, avec une mortalité dépassant 90 p. 100 . D'autre part, si le sang ingéré par les Ornithodores est trop pauvre en microfilaires, de nombreux exemplaires ne s'infestent pas et l'on court le risque de perdre la souche ou de réaliser des expériences illisibles.

Les conditions optima nous paraissent être l'infestation de nymphes d'O. tartakovskyi au deuxième ou troisième stade, gorgées sur un Mérion présentant 5 microfilaires au minimum et 15 au maximum par goutte de sang prélevé à la queue; quelques Ornithodores seront disséqués aussitôt après le repas comme test de la richesse moyenne du sang ingéré. Dans ces conditions, la mortalité chez les Ornithodores ne dépassera guère 20 p. 100.

$$
* *
$$

Chez le Mérion, l'infestation pourra être obtenue soit par piqûre d'Ornithodores, soit par inoculation sous-cutanée de larves au $3^{\circ}$ stade. Cette seconde technique a l'avantage de permettre d'inoculer un nombre de larves connu, compté lors de la dissection de l'Ornithodore, par aspiration dans une pipette Pasteur, d'effilure assez large pour ne pas blesser les larves.

Il faut, quelle que soit la température (hiver ou été), de 40 à 55 jours pour que les larves au $3^{\circ}$ stade introduites chez le Mérion deviennent des adultes ayant atteint leur maturité sexuelle, c'est-àdire pour qu'apparaissent dans le sang de l'animal les microfilaires.

La recherche de ces microfilaires dans le sang du Mérion est malaisée, ces larves passant difficilement dans les capillaires de la queue ; cependant, la vaso-dilatation peut être obtenue par une légère anesthésie générale du Mérion à l'éther, et les microfilaires sont alors aisément comptées dans un volume de sang précis, mesuré à la micropipette graduée. Il n'existe pas de phénomène de périodicité dans la circulation des microfilaires, ni quotidienne, ni saisonnière.

La recherche des filaires adultes à l'autopsie doit être faite avec un très grand soin : les adultes qui vivent dans le tissu cellulaire sous-cutané sont aisément trouvés par dépouillement du Mérion, mais ceux qui se logent entre les aponévroses musculaires profondes et parfois dans les cavités abdominale ou thoracique sont beaucoup plus difficiles à récupérer. Une technique, qui nous a donné de bons résultats, consiste à abandonner le cadavre dilacéré, après 
cette dissection très poussée, dans un grand verre à pied empli d'eau physiologique ; des filaires passées inaperçues à la dissection peuvent être retrouvées dans le résidu de décantation.

La longévité des filaires adultes paraìt très grande : déjà supérieure à seize mois dans nos expériences en cours. Les infestations même très riches ne paraissent causer aucun trouble au Mérion ; nous avons conservé des Mérions parfaitement bien portants, présentant dans le sang un grouillement de microfilaires incomptables : sacrifiés en pleine santé, ces Mérions montraient des infestations supérieures à 250 filaires adultes. Rappelons cependant que de tels Mérions, s'ils présentent un grand intérêt au point de vue des recherches thérapeutiques par exemple, ne peuvent être utilisés pour l'expérimentation avee les Ornithodores, leur taux de microfilaires étant mortel pour ceux-ci.

La transplantation des filaires adultes sur des Mérions neufs est facile et réussit parfaitement : les filaires récoltées chez les Mérions morts ou sacrifiés sont introduites sous la peau des Mérions neufs par une petite boutonnière faite aux ciseaux ou au bistouri et refermée ensuite par une agrafe ou un point de suture. Les microfilaires apparaissent dans le sang des Mérions «transplantés 》 dans les quelques jours qui suivent. Les mèmes filaires adultes ont pu ètre ainsi transplantées quatre fois de suite. Cette technique, outre qu'elle peut servir à conserver les filaires des Mérions morts accidentellement au cours de l'expérimentation, permet de créer artificiellement tous les types d'infestation possibles chez le Mérion, et en particulier de choisir pour chaque expérience ainsi faite le nombre de femelles ou de mâles, ce qui est impossible dans l'infestation de type naturel par piqûre d'Ornithodores ou même par inoculation de larves au $3^{\circ}$ stade, le sexe futur n'étant pratiquement pas distingable à ce stade.

$$
\text { *3\% }
$$

Les avantages offerts par cette filaire, son cycle évolutif et ses hôtes, sur les filaires utilisées jusqu'à présent dans les laboratoires, sont évidents.

Nous ne parlerons pas des filaires de Batracien, sans grand intérêt pratique pour les laboratoires, mais seulement des deux filaires largement utilisées pour l'expérimentation. La première, la filaire du chien, Dirofilaria immitis, a, entre autres défauts, celui d'être transmise par des moustiques dont l'élevage et la manipulation sont fort malaisés et d'exiger un animal d'expérience onéreux et peu maniable. 
La seconde, la filaire du cotton-rat, Litomosoides carinii, outre la difficulté que l'on peut avoir en Europe à se procurer des cottonrats, a l'inconvénient d'avoir comme hôte vecteur un Acarien, Bdellonyssus bacoti, dont il suffit de voir l'importante littérature qui a été consacrée à son élevage, pour se rendre compte combien il est malaisé de le conserver au laboratoire. D'autre part, la petite taille de cet Acarien, sa fragilité, la fréquence nécessaire de ses repas en rendent l'utilisation pour les expériences très méticuleuse et difficile.

L'unique inconvénient que nous avions trouvé à l'utilisation de la filaire du Mérion, c'est-à-dire l'irrégularité du passage des microfilaires dans le sang de la queue, a été résolu, nous l'avons dit, par l'anesthésie légère du Mérion à l'éther.

$$
* *
$$

En résumé, la filaire du Mérion, Dipetalonema blanci, d'une grande longévité chez son hôte vertébré, à localisation le plus souvent sous-cutanée, émettant sans périodicité des microfilaires nombreuses, et dont les formes infestantes peuvent être conservées pendant plus d'un an chez les Ornithodores à jeun, nous semble être pour les laboratoires un matériel de premier choix.

\section{BIBLIOGRAPHIE}

1. Baltazard (M.), Bahmanyar (M.), Pournaki (R.) et Mofidi (Ch.). - Ann. Parasit., 27, 1952, 311.

2. Baltazard (M.), Chabaud (A.-G.) et Minou (A.). - C.R. Acad. Sci., 234, 1952, 2115.

3. Сhabaud (A.-G.). - Ann. Parasit., 27, 1952, 250. 